\title{
THE ECONOMIC THEORY OF INSURANCE
}

\author{
KARL BORCH
}

Bergen

(Notes for an informal discussion in Edinburgh, I June 1964)

\section{Introduction}

1.1. - Under Subject 4 at this Congress we have discussed the practical application of modern statistical techniques in different branches of insurance. During the last decades, there has been an almost explosive development in theoretical statistics and related branches of mathematics. I think it has been very useful to survey the techniques, which have been developed, and find out if they can be used in insurance.

1.2. - There may, however, be some danger in this approach. When new means become available, we should of course have an open mind, and examine these means in order to see if they can serve our ends. We should, however, not get so excited over the power of new techniques, that we distort our ends just for the sake of being able to apply the means.

Linear programming, to take an example, is a powerful tool, which has proved extremeiy useful in many, apparently very different fields. There is, however, little point in using this technique in insurance, unless we have problems which consist of determining the maximum of a linear expression, subject to linear restraints. If there are problems in insurance which can be cast in this form, with sufficient approximation, then linear programming is obviously useful. If, however, we lose something essential by reformulating our problems in this way, linear programming may become a dangerous temptation, which we should resist.

1.3. - In this paper I shall take a different approach. I shall try to take a good and hard look at the ends, with the hope that this will enable us to specify the means which we require. If these means already exist, all is well. If we cannot find any suitable techniques in the mathematician's armoury, we will have to do our own basic research, and develop the tools we need. 
A generation ago, the subject "applied mathematics" consisted mainly of techniques which had proved extremely useful in classical physics. These techniques were used with considerable enthusiasm and little success in economics and other social sciences. The new statistical techniques which excite us to day, have to a large extent been developed to solve problems in quantum mechanics and telecommunications. We may therefore ask ourselves if we have any reason to expect these techniques to be useful in actuarial work.

This point has been made with considerable force by Von Neumann and Morgenstern ([5] p 6) who make the blunt statement: "It is unlikely that a mere repetition of the tricks which served us so well in physics, will do for the social phenomena too". They sum up their view: "It is therefore be to expected-or feared-that mathematical discoveries of a stature comparable to that of calculus will be needed in order to produce decisive success in this field (i.e. economics)".

It is in this spirit we shall try to analyse the ends and means of actuarial science.

\section{The Principle of Equivalence}

2.1. - To illustrate the point which I want to make, we shall begin by discussing an extremely simple example.

Consider an insurance contract under which the only possible payment is an amount of one monetary unit. We shall assume that this amount becomes payable if, and only if an event with probability $p$ should occur.

This contract will define the following claim distribution:

$o$ with probability $\mathbf{I}-p$

I with probability $p$

The net premium of the contract is by definition $p$.

2.2. - We shall next assume that an insurance company offers the contract we have described to the public, at a premium $x>p$. We shall assume that there is a demand for the insurance cover given by this contract, and that demand depends on the premium. We shall formalize this by assuming that the company will be able 
to sell $n=n(x)$ contracts if the premium is set at $x$. It is natural to assume that $n(x)$ will increase with decreasing $x$.

The problem is now to determine the premium $x$ at which the company should offer this insurance contract in the market. This seems to be a very simple problem, and we ought to solve it in a satisfactory manner, before we tackle more complicated problems, or embark on the more ambitious task of constructing a general theory of insurance.

2.3. - In classical theory our simple problem is solved by applying the Principle of Equivalence. According to this principle, the premium should be equal to expected claim payments + administrative costs. This means that $x$ should be determined by

$$
x=p+\frac{\mathbf{I}}{n} C(n)
$$

where $C(n)$ is the cost involved in selling and managing a portfolio of $n$ contracts. If we assume that costs can be split up into "fixed" and "variable" costs, we can write

$$
C(n)=C_{1}+n C_{2}
$$

The premium will then be given by the equation

$$
x=p+C_{2}+\frac{C_{1}}{n(x)}
$$

We have assumed that $n(x)$ decreases with increasing $x$. This means that both sides of the equation will increase with $x$, so that the equation may have any number of solutions, depending on the shape of the function $n(x)$.

2.4. - The Principle of Equivalence gives a neat solution to our simple problem -if we are prepared to disregard the somewhat academic question about existence and uniqueness of the roots of the main equation. To solve the problem in practice, we have to know:

( i) The basic probability $p$

(ii) The cost elements $C_{1}$ and $C_{2}$

(iii) The function $n(x)$

To obtain this knowledge, we will usually have to resort to 
statistical methods, or to be more precise, the techniques of statistical estimation.

The traditional task of the actuary is to provide the best possible estimate of $p$. He is also frequently called upon to supply estimates of $C_{1}$ and $C_{2}$, since this often requires statistical analysis.

The determination of the last element, the function $n(x)$ is usually considered as being outside the duties of the actuary.

In most cases it will probably be the sales manager of the company or a market research department, who is responsible for guessing or estimating the shape of $n(x)$.

2.5. - The function $n(x)$ represents the demand or the market for the insurance contract under consideration. These are economic concepts, and this indicates that our problem cannot be satisfactorily solved, unless we bring in some elements of economic theory.

In some cases it may be possible to determine the "correct" premium without knowing the number of contracts which will be sold. This will be the case if $n(x)$ is approximately constant, or in terms of economics, if insurance has a "low price elasticity". It may seem fairly safe to assume that this actually is the case, if there is no evidence that lower premiums will lead to a significant increase in sales. One should, however, bear in mind that a reduction in the premium or an increase in the agent's commission, come to the same thing for the company, but that they may have very different effects on sales. If we ignore, or "assume away" $n(x)$ in our calculations, we may therefore lose something which is essential to the problem we set out to study.

\section{3. - Operational Research and the Theory of Risk}

3.1. - If an insurance contract is offered to the public at a premium determined by the principle of equivalence, the expected profits on this transaction will be zero. The absence of profits is unpleasant in business, but this is not the point which we want to discuss here.

If an insurance company consistently makes losses on its operations, the company will sooner or later be unable to fulfill its part of the insurance contracts. This means of course that the "insur- 
ance" contracts do no longer serve the very purpose for which they were designed, i.e. to provide almost absolute security to the insured persons.

These considerations indicate that the premium must be set higher than dictated by the principle of equivalence. It is, however, an open question how much higher the premium should be, so that the simple problem discussed in para 2.2. is still unsolved.

3.2. - The simple problem is rarely explicitly formulated in actuarial literature, and no general solutions have been suggested. It is, however, undeniable that the problem exists, and it has not been completely ignored. I think we can distinguish at least three different ways in which authors have tried to attack the problem:

(i) The problem can be dismissed as too simple. It is obvious that the problems we meet in practice are vastly more complicated, and "practical" men may well claim that they have to spend their time solving these more "serious" problems. It is most likely that they have to make their decisions without full knowledge of the true probability $p$ and of the exact shape of the demand function $n(x)$, and that they claim that these decisions are rational or correct. However, if the simple problem is put aside for such reasons, the implications are that the problem becomes easier to solve if we bring in complications, and that ignorance can help us to make the right decisions.

(ii) One can add a safety loading to the premium determined by the principle of equivalence, so that expected profit becomes positive. This idea has probably originated in economic theory, where it is felt that expected profits should be greater the greater the "risks" are. However, economic theory has not so far, been very successful in defining the concept of "risk" and establish its relations to expected profits.

(iii) One can take the probability of ruin as a starting point. In our simple example this means that we consider the probability that the company will suffer a loss if the insurance contract is offered at a premium $x$. This approach is usually taken in actuarial literature; it is often referred to as the Theory of Risk. This theory is in many ways very attractive, but it has found few applications in practice. The reason is-I believe-that the theory does not 
come to grips with the real problems as practising actuaries see -or feel-them.

3.3. - We shall now try a different approach to the problem, and in doing so we shall ignore the cost elements. This involves no loss of generality, since these elements can be brought in explicitly at any stage in the argument.

If an insurance company has underwritten $n$ contracts against a premium $x$, the outcome can be any result between the two extremes:

(i) A loss of $n(\mathrm{I}-x)$, if all contracts lead to a claim.

(ii) A profit of $n x$, if no claims are made.

In general the profit $z$ (positive or negative) will have a probability distribution determined by:

$$
\operatorname{Pr}(z \leqslant n x-y)=\sum_{j \geqslant y}\left(\begin{array}{l}
n \\
j
\end{array}\right) p^{j}(\mathrm{I}-p)^{n-j}
$$

where $n$ depends on $x$.

3.4. - From these considerations we see that the decision to offer an insurance contract to the public at a premium $x$ will give the company a profit which is a stochastic variable. The probability distribution of this variable will depend on $x$, the claim distribution and the demand function. This means that the choice of a market premium $x$ implies the choice of a profit distribution.

If we now assume that an insurance company has some rules which enable it to decide on the premium at which the contract should be offered, it must also have a rule which makes it possible to pick out the best or the most preferred among the obtainable profit distributions. This rule will represent the company's willingness to assume risk, or its risk policy, or to use still another term, the objectives, which the company wants to pursue.

3.5. - The choice of policy or objectives is by its very nature a subjective decision. It is not possible to state categorically that it is right or wrong if the company underwrites a given risk. It may, however, be possible to state whether a particular underwriting decision is consistent or not with the overall objectives of the company. 
In order to formalize these ideas, we shall assume:

(i) An insurance company has a complete preference ordering over the set of all profit distributions.

This ordering will represent the company's policy, and in every situation the company will seek to make the decision which leads to the most preferred among the attainable profit distributions.

(ii) The company's preference ordering is consistent.

This term obviously requires a precise definition, a point which we shall not take up here. The different possible definitions have been studied in detail by a number of authors, i.a. Savage [6], and the application to insurance has been discussed in another paper [2].

3.6. From these assumptions it follows trivially that it is possible to assign a real number or an index $U\{F\}$ to any profit distribution $F(z)$ so that

$$
U\{F\}>U\{G\}
$$

if and only if $F(z)$ is preferred to $G(z)$.

It follows further that there exists a real valued function $u(z)$ such that

$$
U\{F\}=\int_{-\infty}^{+\infty} u(z) d F(z)
$$

This result is far from trivial. It was first proved by Von Neumann and Morgenstern [5] in 1947. Since then a number of other proofs have been published, for instance in the book by Savage [6] already referred to.

The implication of this result to our problem is that any consistent rule for determining the premium for an insurance contract can be represented by a function $u(z)$. This function is usually referred to as a utility function, because it can be interpreted as the utility assigned to an amount of money equal to $z$. The concept "utility of money" plays a central part in classical economic theory, and it is interesting to note that this concept also appears necessary for further development of the theory of insurance.

3.7. - In order to illustrate the application of the ideas, developed in the preceding paragraph, we shall study an example, slightly less trivial than the one introduced in para 2.I. We shall find it convenient to make some changes of notation. 
We shall consider an insurance company and assume:

i) The company's policy can be represented by a utility function $u(x)$.

(ii) The company's initial capital (or free reserves) is $S$.

(iii) The company considers offering the public an insurance contract with a claim distribution $F(x)$.

(iv) The premium for this contract is fixed as $P$, for instance by tariff-agreement or Government regulation.

(v) If the company spends an amount $s$ on advertising and sales promotion, it will be able to sell $n=n(s)$ contracts.

The problem is then to determine the optimal amount $s$ which should be spent on sales promotion.

By a straight forward application of the results in para 3. 6. we find that $s$ should be determined, so that the following expression is maximized:

$$
\int_{0}^{\infty} u(S+n P-s-x) d F^{(n)}(x)
$$

where $F^{(n)}(x)$ is the $n$-th convolution of $F(x)$ with itself.

This value of $s$ will lead to the profit distribution which according to the company's policy, is considered the best attainable.

3.8. - In the example above we have reformulated our original Problem so that our task finally was reduced to maximizing a mathematical expression. This approach to a problem is typical of Operational Research. This term is often used loosely about a group of more or less interrelated mathematical techniques. However, the essential idea, and the real art of operational research lies, not in solving a particular class of mathematical problems, but in formulating the problem so that these mathematical techniques can be applied.

3.9. - If the formulation given in para 3.7. comes to grips with the problem as practising actuaries see it, they should be able to decide in general terms on the kind of mathematical techniques which are required to solve the problem. The choice of specific techniques can probably best be made in each particular case, depending on the nature of the three functions $u(x), n(s)$ and $F(x)$. Of these three functions, $F(x)$ is well known to any actuary, and 
$n(s)$ represents a concept which should be familiar to company actuaries who keep in contact their colleagues in the market research department. The utility function $u(x)$ may, however, seem strange and unfamiliar to many actuaries. The function represents the company's policy, and so far, little is known about the general shape of these utility functions. The main reason for this lack of knowledge is that few companies are very specific when they make public statements about their policy. This may mean that companies simply do not have a well-defined policy. It may, however, also mean that companies consider their policy a business secret. The companies may have good reasons for doing this. For instance in negotiations over a reinsurance treaty, it must be important for a company to hide that its real policy is to obtain cover almost at any cost.

We shall not pursue this subject any further. The possible shape of the utility function is discussed in some detail in another paper $[\mathrm{I}]$, and the problem has recently been studied by Welten [7].

3.10. - Our formulation does of course oversimplify the real problem, and this may mean that we have lost something which is essential-or to put it another way-that we have solved the wrong problem-a problem which cannot occur in practice.

The two most serious aspects of our simplifying assumptions appear to be:

(i) We have studied an isolated decision to be taken once and for all. This means that we have ignored any implications the decision may have on the future of the company.

To meet objections on this point, we can formulate the problem in terms of a dynamic model. An attempt in this direction has been made in another paper [4].

(ii) We have assumed that the company was alone in the market, or that our company reached its decision without considering the decisions or actions which competing companies might take.

We shall discuss this point in the following chapter, and we shall see that this leads us towards an economic theory of insurance.

\section{Risk and Economic Theory}

4.1. - In para 3.7. we assumed that there existed a function $n(s)$ 
which determined the number of insurance contracts $n$ which our company could sell if an amount $s$ was spent on sales promotion. This function represented the market situation which confronted the company.

If more than one company operates in the market, the situation cannot be represented by a single function of one variable. If there are $k$ companies, we may get an adequate description of the situation by specifying $k$ functions

$$
n_{i}\left(s_{1} \ldots s_{k}\right) \quad(i=\mathrm{r}, 2 \ldots k)
$$

Here $n_{i}$ is the number of contracts which company $i$ will sell if the $k$ companies spend the amounts $s_{1} \ldots s_{i} \ldots s_{k}$ to promote their sales.

4.2. - In this model the task of company $i$ will still be to maximize a mathematical expression of the same form as the one we considered at the end of para 3.7. However, this expression will now depend on the $k$ variables $s_{1} \ldots s_{k}$, and company $i$ controls only one of these. The remaining $k-\mathbf{I}$ variables are controlled by the other companies, and they will seek to use this control to pursue objectives which may be different, and even directly opposed to those of the company under consideration. This means that company $i$ cannot select an optimal $s_{i}$ without knowing or guessing the values which the other companies will select for $s_{1} \ldots s_{i-1}$, $s_{i+1} \ldots s_{k}$. These other companies will, however, be in exactly the same kind of dilemma, so the whole situation becomes a game as to who can outguess whom.

4.3. - It is obvious that the situation we have described is essentially different and more complicated than the situations which we analysed in chapter 3 . It is also obvious that the companies in this situation cannot reduce their problems to the simple maximizing problem considered in para 3.8. Such a reduction of the problem is the very essence of the approach which leads to operational research. If this reduction is impossible, we must look for a different approach.

4.4. - The situation we have described is not very different from the classical model of a market where several sellers or producers compete for the favour of a large number of buyers or consumers. 
Classical economic theory has been able to analyse such markets in a rather satisfactory manner, and it is natural to try if this theory can provide an approach which leads to a solution to our problem.

This leads us to consider insurance cover as a commodity for which there is a demand, depending on the price. We must then assume that some persons or institutions are willing and able to supply this commodity, and that the amount they will supply depends on the price.

If the supply and demand functions meet certain conditions, there exists a unique price which will make total supply in the market equal to total demand.

This price is referred to as the equilibrium price.

4.5. - The basic assumption of the classical market theory is that the traders behave in a passive manner, in the sense that they take the price as given and unchangeable, and decide how much they want to buy or sell at this price. If the traders make their decisions on the basis of a price different from the equilibrium price, supply and demand will be unequal, and this will generate forces which push the price towards the equilibrium price.

The crowning achievement of the classical theory was to prove that if all traders made their decisions on the basis of the equilibrium price, the market would reach a Pareto-optimal state. This means roughly that the market is in a state where no trader can improve his situation, except at the expense of others. This means that the price mechanism establishes a rational arrangement in a market which initially seemed to be a chaos of conflicting interests. This again led classical economists to claim that free competition would lead to "the best of all possible worlds".

4.6. - If we try to apply the ideas of classical economic theory to an insurance market, we will run into difficulties almost immediately. One of the first difficulties is that there is no natural unit of insurance cover, so that it seems impossible to define price in a meaningful way. There are other difficulties of an even more fundamental nature, but I shall not deal with them here, since they have been discussed in detail in another paper [3].

Even if many of the basic concepts of classical economic theory 
are meaningless or inapplicable in an insurance market, the most fundamental of them all, Pareto-optimality, can be defined fairly easily. It is therefore natural to take this concept as our starting point. This leads us to the Theory of Games [5], which in this context must be seen as a far-reaching generalization of the more orthodox economic theories.

4.7. - The basic assumption in the theory of $n$-person games is that rational players will somehow come to a Pareto-optimal arrangement. This leads to another difficulty, since there usually will be an infinity of such arrangements.

To illustrate this, we can again consider the example of paras 4.I.-4.2. In this example there may well be a unique advertising expenditure which will be optimal for the $k$ companies, seen as a group. There will, however, be infinitly many ways in which this expenditure and its fruits can be divided among the $k$ companies.

To obtain a determinate solution to such problems, we must make additional assumptions about how the parties behave during negociations or bargaining. In game theory such behavioral assumptions concern the ways in which the players form coalitions in order to co-operate during the negociations towards a Pareto-optimal arrangement.

Classical economic theory reached a determinate solution, i.e. a unique equilibrium price by making the "additional assumption" that traders passively adjusted to prices, as if they were given by some deus ex machina. This assumption may be realistic or not, the point in the present context is that it has no meaning when applied to an insurance market. We must find other assumptions of about the same strength if we want to treat insurance as an economic activity, and analyse it within the framework of a general economic theory.

\section{Concluding Remarks}

5.1. - The point I have tried to make in this paper, is that the ends should guide our choice of means. We should not adjust the ends in order to create new applications for means which happen to be fashionable.

A good actuary should of course explore new mathematical techniques and find out if they can be of help in his work. I do, 
however, not believe that this is the most pressing need, neither in the actuarial profession nor in the insurance industry.

5.2. - In chapter 3 I have tried to show that the methods of operational research can be successfully applied only in insurance companies which have a well-defined policy-or to put it tautologically-companies which can spell out their objectives in an operational manner.

In chapter 4 I have indicated that there are situations in which the methods of operational research fall short. Mathematical methods which may prove useful in these situations, have been developed in game theory. The methods appear powerful, but we cannot hope to use them successfully unless we are quite clear about the objectives--the objectives of persons and companies, when they act individually, and when they act in groups where the members have partially conflicting interests.

5.3. - The stress on objectives really means that we need more factual knowledge before we start experimenting with new mathematical techniques. We need to know more about man's need for security and willingness to take risks before we devise the insurance which will solve his problems.

It may be fitting to terminate this paper by quoting the conclusions. Von Neumann and Morgenstern reached in their analysis of the application of mathematical methods in economic theory: "The underlying vagueness and ignorance has not been dispelled by the inadequate and inappropriate use of a powerful instrument that is very difficult to handle" ([5] pp 4-5).

\section{REFERENCES}

[I] Borch, K.: Reciprocal Reinsurance Treaties. The ASTIN Bulletin, Vol. I, pp. I 70-I9I.

[2] Borch, K.: The Utility Concept Applied to the Theory of Insurance. The ASTIN Bulletin, Vol. I, pp. 245-255.

[3] Borch, K.: Equilibrium in a Reinsurance Market. Econometrica, Vol. 3o, pp. 424-444.

[4] Borch, K.: Payment of Dividend by Insurance Companies. Transactions of the I 7 th International Congress of Actuaries, Vol. III, pp. 527-540.

[5] Neumann, J. von and O. Morgenstern: Theory of Games and Economic Behavior, 2nd Edition, Princeton 1947.

[6] Savage, L. J.: The Foundations of Statistics, New York I954.

[7] Welten, C. P.: Reinsurance Optimization by Means of Utility Functions, Actuariële Studiën, February 1964, pp. I66-I75. 